\title{
Phase II evaluation of mitoxantrone in advanced pancreatic carcinoma: A Southwest Oncology Group Study
}

Sarah A. Taylor ${ }^{1}$, Thomas Fleming ${ }^{2}$, Daniel D. Von Hoff ${ }^{3}$, Joseph D. McCracken ${ }^{4}$, Ronald M. Bukowski ${ }^{5}$, Robert W. Talley ${ }^{6}$, Robert B. Natale ${ }^{7}$, J.T. Guy ${ }^{8}$, Wolfram E. Samlowski ${ }^{9}$ and John H. Costanzi ${ }^{10}$ ${ }^{1}$ University of Kansas Medical Center; ${ }^{2}$ Southwest Oncology Group Biostatistical Center - Seattle;

${ }^{3}$ University Texas Health Science Center - San Antonio; ${ }^{4}$ Brooke Army Medical Center; ${ }^{5}$ Cleveland Clinic Foundation; ${ }^{6}$ Henry Ford Hospital; ${ }^{7}$ University of Michigan Medical Center; ${ }^{8}$ Ohio State University Hospital; ${ }^{9}$ University of Utah Medical Center; ${ }^{10}$ University of Texas - Galveston, USA

Key words: mitoxantrone, pancreatic carcinoma, phase II trial, DHAD, novantrone

\section{Summary}

Patient with advanced adenocarcinoma of the pancreas and no prior chemotherapy were treated on a Phase II trial of mitoxantrone. Doses were adjusted for hepatic dysfunction as defined by bilirubin. Twenty-four patients with a bilirubin $\leq 1.5 \mathrm{mg} \%$ received mitoxantrone $12 \mathrm{mg} / \mathrm{m}^{2}$ i.v. repeated every three weeks. Myelosuppression in the form of leukopenia was the major toxicity. There were no responses in twenty-four evaluable patients.

\section{Introduction}

Mitoxantrone (Novantrone; dihydroxyanthracenedione) is one of the anthracenedione derivatives synthesized in an attempt to find an agent with the same or better antitumor activity than adriamycin without its dose-limiting cardiotoxicity $[1,2]$. Phase I studies showed mitoxantrone to be well tolerated with reversible myelosuppression as its dose-limiting toxicity [3]. Its antitumor activity has been similar to adriamycin.

In order to insure an appropriate distribution of untreated patients on Phase II trials in advanced pancreatic cancer, the Southwest Oncology Group initiated a series of randomized Phase II trials. We report a trial of mitoxantrone in advanced adenocarcinoma of the pancreas.

\section{Materials and methods}

Previously untreated patients were randomized to a combination of fluorouracil, adriamycin, mitomycin and streptozotocin (FAM-S) or to mitoxantrone as a single agent. At relapse or disease progression patients who still met eligibility requirements were reregistered and crossed over to the other arm.

Patient eligibility included histologic confirmation of adenocarcinoma of the pancreas not amenable to curative surgery or radiation; objective measurable disease; a life expectancy of at least six weeks; a performance status of three or better by SWOG criteria; WBC count $\geq 3500 / \mathrm{mm}^{3}$; a platelet count of $\geq 125,000 \mathrm{~mm}^{3}$, a serum creatinine $\leq$ $2.0 \mathrm{mg} \%$. There were no age restrictions. Patients had received no chemotherapy except at cross-over. At cross-over patients had recovered from any acute toxicities and had received no chemotherapy for six weeks. Patients with past or present history of congestive heart failure were not eligible. In pa- 
tients with recent abdominal surgery, treatment was held for 3 weeks or until recovery of bowel function, whichever occurred later. Concomitant radiation therapy was allowed for painful boney metastases only. Patients were stratified by performance status $(0-1$ vs $2-3)$ and according to whether biopsy only or palliative bypass procedures were performed.

During treatment, weekly complete blood counts, WBC and differentials, and platelets were monitored. Tumor measurements, kidney and liver function tests, EKG's, and performance status were obtained prior to each course.

Cardiac ejection fractions determined by gated radionuclide angiography were obtained in all patients pretreatment and monitored thereafter in those patients who had received their maximal dose of adriamycin or who had received mitoxantrone for six weeks or more.

Doses of mitoxantrone were adjusted for hepatic function. The initial dose of mitoxantrone for patients with a bilirubin $\leq 1.5 \mathrm{mg} \%$ (good risk) was $12 \mathrm{mg} / \mathrm{m}^{2}$ given as a 30 minute infusion in $100 \mathrm{cc}$ of $\mathrm{D}_{5} \mathrm{~W}$ through a freely running intravenous line. Patients with an abnormal bilirubin were considered as poor risk and treated with reduced doses. Patients with a bilirubin between $1.5-3.0 \mathrm{mg} \%$ received $6 \mathrm{mg} / \mathrm{m}^{2}$ and patients with a bilirubin of greater than $3 \mathrm{mg} \%$ received $4.5 \mathrm{mg} / \mathrm{m}^{2}$. The treatment was repeated every three weeks provided the patient had recovered from previous drug induced toxicity. Doses for subsequent courses were adjusted based upon previous drug induced toxicity. Patients without side-effects (WBC $\geq 4000 / \mathrm{mm}^{3}$, Platelets $\geq 100,000 / \mathrm{mm}^{3}$ ) had their doses increased by $10 \%$. Patients with WBC $2000-3900 /$ $\mathrm{mm}^{3}$ or platelets $50,000-90,000 / \mathrm{mm}^{3}$ which had resolved at 3 weeks received the same dose. Patients with WBC $1000-1900 / \mathrm{mm}^{3}$ or platelets $25,000-$ $49,900 / \mathrm{mm}^{3}$ or requiring more than 1 week to recover had their dose reduced by $17 \%$. Patients with life threatening toxicity (WBC $<1000 / \mathrm{mm}^{3}$, platelets $<25,000 / \mathrm{m}^{3}$ ) had their dose reduced by $50 \%$.

Response and survival duration were calculated from the initial date of treatment. Complete remission was defined as complete disappearance of all
Table 1. Patient characteristics of previously untreated patients

\begin{tabular}{lcc} 
& Good risk & Poor risk \\
\hline No. entered & 24 & 5 \\
Evaluable & 24 & 5 \\
P.S.: Median & 2 & 2 \\
P.S.: Range & $0-3$ & $0-3$ \\
Age: Median & 54 & 66 \\
Age: Range & $27-82$ & $34-70$ \\
Biopsy only & 19 & 3 \\
Diversion procedure & 5 & 2 \\
Courses: & & \\
$\quad$ Administered & 62 & 6 \\
$\quad$ Median & 2 & 1 \\
$\quad$ Range & $1-10$ & $1-2$ \\
Response: & & \\
$\quad$ Progression & 19 & 4 \\
$\quad$ Early death & 2 & 0 \\
$\quad$ Stable & 3 & 1.4 \\
Survival (months): & & \\
$\quad$ Median & 3.1 & \\
\hline
\end{tabular}

clinical evidence of tumor for a minimum of four weeks. Partial remission was defined as $50 \%$ or greater decrease in the sum of the products of all diameters of measured lesions with no new disease or increase in size of any lesion. Progression was defined as the unequivocal increase of at least $25 \%$ in the size of any measurable lesion or the appearance of new lesions.

\section{Results}

Thirty-six patients were registered on study. All patients were eligible and all were evaluated. Patient characteristics are presented in Table 1.

Twenty-four previously untreated good risk patients were evaluable and received mitoxantrone at $12 \mathrm{mg} / \mathrm{m}^{2}$. Three patients had stable disease for 73 , 195 and 233 days. Twenty-one patients had progressive disease with two early disease related deaths (days 3 and 8).

Five untreated poor risk patients received reduced doses of mitoxantrone ( 3 at $4.5 \mathrm{mg} / \mathrm{m}^{2}$ level and 2 at $6.0 \mathrm{mg} / \mathrm{m}^{2}$ level). There were no objective responses. There was one early death due to disease at day 14. The survival curves for these two groups of patients (good and poor risk previously untreated) are shown on Fig. 1. 


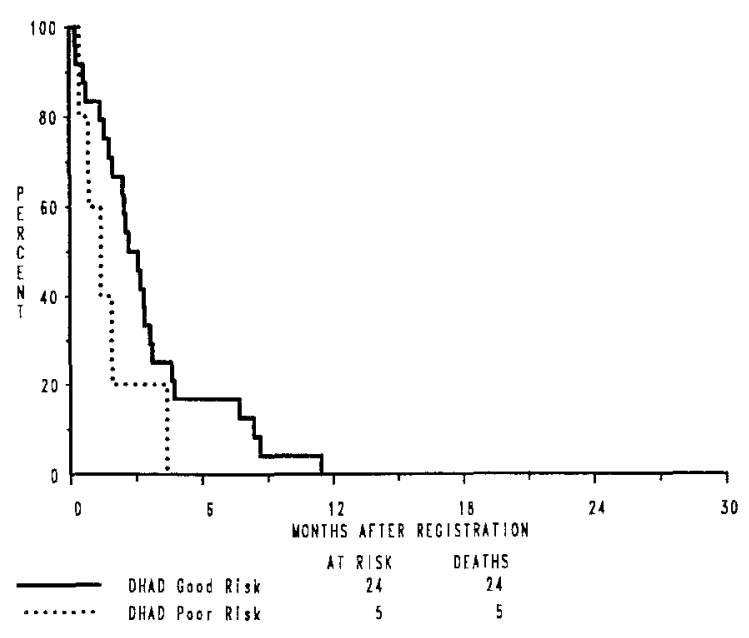

Fig. 1. Overall survival eligible patients with follow-up.

Seven patients were randomized to mitoxantrone after failing FAM-S. Three good risk patients received eight courses at $12 \mathrm{mg} / \mathrm{m}^{2}$. One patient treated with $6 \mathrm{mg} / \mathrm{m}^{2}$ was lost to follow up and the second patient died day seven due to disease. One patient was treated with $4.5 \mathrm{mg} / \mathrm{m}^{2}$ and expired after one course. One patient was treated with only $3 \mathrm{mg} / \mathrm{m}^{2}$ due to dose reduction error. She expired on day three. There were no objective responses.

\section{Toxicity}

Myelosuppression in the form of leukopenia was the major toxicity in the good risk group. Forty-two courses at the $12 \mathrm{mg} / \mathrm{m}^{2}$ dose were evaluable for myelosuppression. The median leukocyte nadir for this group was $2300 / \mathrm{mm}^{3}$ (Range $500->20,000$ ). The median platelet nadir was $>150,000 / \mathrm{mm}^{3}$. One patient with concomitant sepsis had a platelet count of $24,000 / \mathrm{mm}^{3}$. Drops in hemoglobin (1-3 grams) without documented bleeding were seen in 5 patients. Two patients required dose reductions of $10 \%$ in seven courses. Dose escalation by $10 \%$ was required in two patients. Leukocyte counts of 2,999 (1000 granulocytes) or less were observed in $72 \%$ of the courses. Mild nausea, vomiting and alopecia were observed. There were no treatment related deaths.
Myelosuppression was minimal in the poor risk patients treated. Two courses of six were not evaluable for hematologic toxicity due to early death. The median leukocyte nadir was $>4000 / \mathrm{mm}^{3}$. The platelet nadir was $\geq 100,000 / \mathrm{mm}^{3}$. One patient had an unexplained 2 gram drop in hemoglobin. Two patients had mild nausea and vomiting.

In the cross-over group receiving mitoxantrone as second line therapy, toxicity was poorly documented. Ten courses were not evaluable for hematologic toxicity (one patient was lost to follow up, and there were three early deaths). In the two courses for which hematologic data was available, there was no myelosuppression.

Cardiac toxicity was difficult to assess due to the limited number of patients who received significant cumulative doses. Five good risk patients received four or more courses of mitoxantrone with no change in ejection fractions by gated radionuclide angiography. One patient experienced an episode of paroxysmal atrial tachycardia between courses three and four. This was not temporally related to her treatment and did not require hospitalization. There was no other clinical or EKG evidence of cardiotoxicity.

\section{Conclusion}

Mitoxantrone was well tolerated, but showed no objective responses in this group of patients, which included twenty-four previously untreated individuals who received adequate doses of drug. The $95 \%$ confidence level for this group of patients is $0-14.3 \%$. We conclude that mitoxantrone, administered at $12 \mathrm{mg} / \mathrm{m}^{2}$ every 3 weeks has no activity in advanced adenocarcinoma of the pancreas.

\section{Acknowledgements}

This investigation was supported in part by the following PHS Cooperative Agreement grant numbers awarded by the National Cancer Institute, DHHS: CA-12644, CA-22433, CA-04919, CA-27057, CA-04920, CA-13238, CA-03096, CA-37429, CA-20319, CA-32734, CA-28862, 
CA-35995, CA-21116, CA-36020, CA-03389, CA-16385, CA-12213, CA-32102.

\section{References}

1. Zee-Cheng RKY, Cheng CC: Anti-neoplastic agents. Structure activity relationship study of bis (substituted aminoalkyeamine) anthraquinones. J Med Chem 21:291- 294, 1979

2. Johnson RK, Zee-Cheng RKY, Lei WW, Action Henry DW,
Cheng CC: Experimental antitumor activity of aminoanthraquinones. Cancer Treat Rep 63:425-439, 1979

3. Von Hoff DD, Pollard E, Kuhn J, Murray E, Coltman Jr CA: Phase I clinical investigation of 1,4 dihydroxy-5-8-bis ([2-([2-hydroxyethyl) amino] ethyl)] amino 9, 10-anthracenedione dihydrochloride) (NSC 301739, DHAD) A New Anthracenedione. Cancer Res 40:1516-1518, 1980

Address for offprints: Southwest Oncology Group (SWOG8043), Operations Office, 5430 Fredericksburg Road, Suite \#618, San Antonio, TX 78229, USA 